ARTICLE

https://doi.org/10.1038/s41467-019-09507-0

\title{
Structural insight into YcbB-mediated beta-lactam resistance in Escherichia coli
}

\author{
Nathanael A. Caveney (10 ${ }^{1}$, Guillermo Caballero', Henri Voedts ${ }^{2}$, Ana Niciforovic ${ }^{1}$, Liam J. Worrall', \\ Marija Vuckovic ${ }^{1}$, Matthieu Fonvielle ${ }^{2}$, Jean-Emmanuel Hugonnet ${ }^{2}$, Michel Arthur (D) $^{2} \&$ Natalie C.J. Strynadka ${ }^{1}$
}

The bacterial cell wall plays a crucial role in viability and is an important drug target. In Escherichia coli, the peptidoglycan crosslinking reaction to form the cell wall is primarily carried out by penicillin-binding proteins that catalyse D,D-transpeptidase activity. However, an alternate crosslinking mechanism involving the L,D-transpeptidase $Y c b B$ can lead to bypass of D,D-transpeptidation and beta-lactam resistance. Here, we show that the crystallographic structure of $Y c b B$ consists of a conserved L,D-transpeptidase catalytic domain decorated with a subdomain on the dynamic substrate capping loop, peptidoglycan-binding and large scaffolding domains. Meropenem acylation of $Y c b B$ gives insight into the mode of inhibition by carbapenems, the singular antibiotic class with significant activity against L,Dtranspeptidases. We also report the structure of PBP5-meropenem to compare interactions mediating inhibition. Additionally, we probe the interaction network of this pathway and assay beta-lactam resistance in vivo. Our results provide structural insights into the mechanism of action and the inhibition of L,D-transpeptidation, and into YcbB-mediated antibiotic resistance.

\footnotetext{
${ }^{1}$ Department of Biochemistry and Molecular Biology and the Centre for Blood Research, University of British Columbia, Vancouver V6T 1 Z3 BC, Canada. ${ }^{2}$ Sorbonne Université, Sorbonne Paris Cité, Université Paris Descartes, Université Paris Diderot, INSERM, Centre de Recherche des Cordeliers, CRC, 75006 Paris, France. Correspondence and requests for materials should be addressed to N.C.J.S. (email: ncjs@mail.ubc.ca)
} 
T he bacterial cell wall plays a critical role in the strength and viability of bacteria within their natural environs. Underscored by the development of beta-lactam antibiotics such as penicillins and cephalosporins, biosynthesis of the major structural component of the bacterial cell wall, peptidoglycan (PG), has been long recognized as an important drug target. PG is composed of extended polymerized glycan strands of alternating beta-1,4 linked $N$-acetyl-glucosamine- $N$-acetyl-muramic acid which crosslink into a net-like mesh via short peptide segments covalently attached at the $\mathrm{C} 3-\mathrm{OH}$ position of the latter sugar ${ }^{1}$. The precursor subunits of PG are synthesised in the cytosol and transferred to an undecaprenyl phosphate lipid carrier ${ }^{2}$. This moiety is then flipped to the periplasmic face of the membrane, where polymerisation into glycan strands, release from lipid carrier and peptide crosslinking to the existing PG sacculus occur in concert ${ }^{2}, 3$.

In Escherichia coli, this peptide crosslinking reaction is primarily carried out by class A and B penicillin-binding proteins (PBPs). These proteins have D,D-transpeptidase activity and catalyse the cleavage of a D-Ala ${ }^{4}-\mathrm{D}-\mathrm{Ala}^{5}$ peptide bond of the acyl donor and subsequent crosslinking of the $\mathrm{D}-\mathrm{Ala}^{4}$ carbonyl to the primary amine of a diaminopimelic acid (DAP) residue on the acceptor. This results in a D-Ala ${ }_{\text {donor }}-\mathrm{DAP}_{\text {acceptor }}^{3}$ crosslink ${ }^{1}$. This D,D-transpeptidase activity can be blocked by beta-lactam antibiotics, which act as a substrate mimetic of the donor strand peptide bond. Inhibition of this final and crucial step in PG biosynthesis results in destabilisation of the cell wall and ultimately cell death ${ }^{4}$.

Despite the success of beta-lactam antibiotics in the clinic, it has recently been shown that an alternate crosslinking mechanism can lead to the bypass of PBP mediated D,D-transpeptidation. The L,D-transpeptidase YcbB (alternately named LdtD), with remarkably few additional accessory factors, is able to undertake this recovery of PG crosslinking function, even in the presence of many clinically used beta-lactam antibiotics. The factors required include the upregulation of alarmone (ppGpp) synthesis, the class $\mathrm{C}$ monofunctional PBP5, and an associated glycosyltransferase (GTase) activity attributed to the class A bifunctional $\mathrm{PBP}^{2} \mathrm{~b}^{5}$ which harbours both GTase and TPase activities in distinct active sites. As with D,D-crosslinked PG, the bypass pathway initiates with GTase catalyzed polymerisation of PG; the resultant growing product strand is acted upon by PBP5 which catalyses a carboxypeptidase reaction targeting removal of the terminal $\mathrm{D}-\mathrm{Ala}^{5}$ resulting in a donor glycan-tetrapeptide substrate for YcbB. In the last step of the bypass pathway, YcbB catalyses L,D-transpeptidation using this tetrapeptide-containing donor to form a $\mathrm{DAP}^{3}{ }_{\text {donor }}-\mathrm{DAP}^{3}$ acceptor crosslink $^{5}$. L,D-transpeptidases, including $\mathrm{YcbB}$, are not efficaciously inactivated by beta-lactams ${ }^{5}$. The formation of a thioester-containing L,Dtranspeptidase-beta-lactam adduct is slow for penicillins and cephalosporins $^{6}$. The acylation reaction is followed by hydrolysis, thereby preventing full inactivation of the enzyme ${ }^{6}$. The D,Dcarboxypeptidase PBP5, is inhibited only at high concentrations of beta-lactams ${ }^{7}$.

Although YcbB is resistant to most beta-lactam antibiotics, it is susceptible to inhibition by carbapenem antibiotics such as meropenem and imipenem ${ }^{5}$. Carbapenem antibiotics are a subset of beta-lactam antibiotics which have previously been shown to inhibit other L,D-transpeptidases ${ }^{8}$. This class of antibiotic is of interest, not only due to its ability to inhibit $\mathrm{YcbB}$, but as well the carboxypeptidase activity of PBP5 and the D,D-transpeptidase activity of PBP1b. Further insight into the inhibition of the enzymes in the D,D-transpeptidase bypass pathway could lead to the development of novel inhibitors to better address the current beta-lactam resistance crisis. Additionally, it was recently shown that copper can inhibit L,D-transpeptidation of bacterial cell walls and specifically inhibit $\mathrm{YcbB}^{9}$. This plays an important role in the inherent antimicrobial activity of copper.

In this work, we report the structure of $E$. coli YcbB acylated with meropenem. YcbB is seen to consist of a conserved L,D-transpeptidase catalytic domain, with the notable additions of a subdomain on the substrate capping loop, a PG binding domain, and a large scaffolding domain potentially important in mediating partner interactions. This complex domain architecture is unique in comparison to that of well characterised Gram positive and $\mathrm{Myco}$ bacterium L,D-transpeptidases. In addition, we report in parallel, the structure of a PBP5-meropenem acyl enzyme complex in order to completely describe the molecular interactions which facilitate meropenem inhibition of the YcbB and PBP5 partners. Our results provide structural insight into D,D-transpeptidase bypass in E. coli and structural insight into the role of L,D-transpeptidation of PG in Gram negative bacteria. Additionally, we probe the protein interaction network and affinities for the D,D-transpeptidase bypass pathway and assay YcbB mediated beta-lactam resistance in an in vivo setting allowing further mechanistic insight into this drug resistance pathway and generated phenotype.

\section{Results and discussion}

E. coli YcbB X-ray crystallographic structure. To investigate the role of YcbB in beta-lactam resistance at the atomic level and provide insight into L,D-transpeptidase activity in Gram negative bacteria, the X-ray crystallographic structure of $E$. coli YcbB was pursued. Cocrystals, generated in the presence of $1 \mathrm{mM}$ meropenem at $\mathrm{pH} 7.5$, displayed $\mathrm{P}_{3} 2_{1} 2$ symmetry with unit cell dimensions of $a=126.5 \AA, b=126.5 \AA, c=88.8 \AA$ and a diffraction resolution of $2.76 \AA$. There is one molecule of $\mathrm{YcbB}$ in the asymmetric unit. The structure solution was phased using a distinct SIR dataset of a heavy atom (ethyl mercury phosphate) soaked crystal with final refinement statistics presented in Supplementary Table 1 . The resulting maps showed well resolved electron density for the majority of the enzyme chain (Supplementary Fig. 1), allowing near complete tracing of the YcbB model (see Methods). As in solution, the structure indicates a monomeric form of the enzyme with no obvious crystallographic formation of larger oligomers. The overall dimensions of the enzyme are $74 \times 73 \times 40 \AA$ with a total surface area of $\sim 24,400 \AA^{2}$, and with a largely positive electrostatic distribution across the catalytic domain and largely apolar distribution across the PG and scaffolding domains. An extended electropositive active site demarcated by bound meropenem antibiotic lies across the catalytic domain with appropriate clefts for both donor and acceptor substrates adjacent and continuous (Fig. 1). The observed overall architecture of YcbB (Fig. 1) has several distinct features from all currently deposited L,D-transpeptidase structures, as suggested from the unique insertions and substitutions in sequence alignments with previously characterized Gram positive and mycobacterial species $(7.1-14.3 \%$ sequence identity; Supplementary Fig. 2). The well ordered central catalytic domain, comprised of residues 375-576, forms a canonical L,D-transpeptidase fold harbouring an extended active site cleft with the expected conserved active site motif ${ }^{10}\left(\mathrm{HX}_{15-18}[\mathrm{~S} / \mathrm{T}] \mathrm{XGCh}[\mathrm{R} / \mathrm{N}]\right.$, where $\mathrm{X}$ represents any residue, and $h$ is any hydrophobic residue), but with the notable insertion of a unique substrate capping subdomain (residues 422-495), unprecedented in size and observed secondary structural elements and lying adjacent to the meropenem-acylated catalytic site. On the opposite face of the central catalytic domain, an also unprecedented N-terminal region, central helix, and C-terminal helical tail (residues 37-233, 352-374, and 605-615 respectively) are proximal and form a collective helical bundle we term the scaffold domain, and predict to play a potential role in protein-protein interactions in $\mathrm{YcbB}$ - 
a
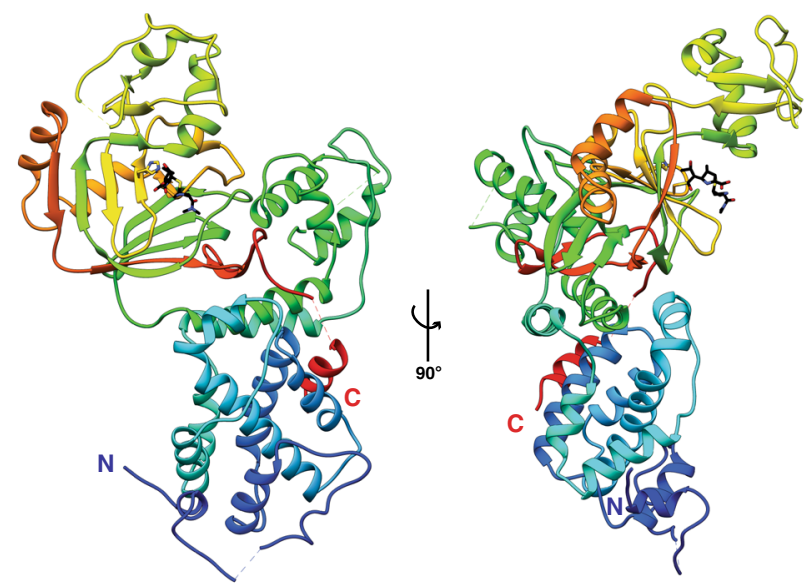

b
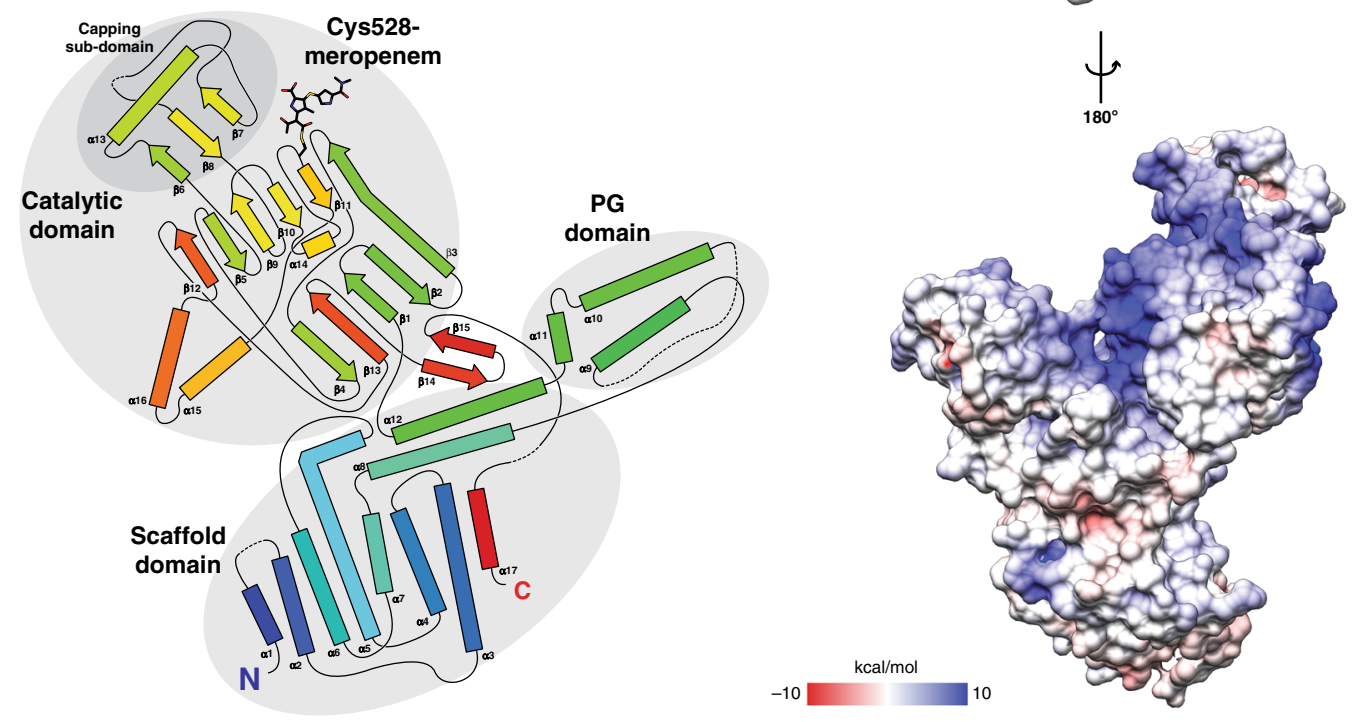

c

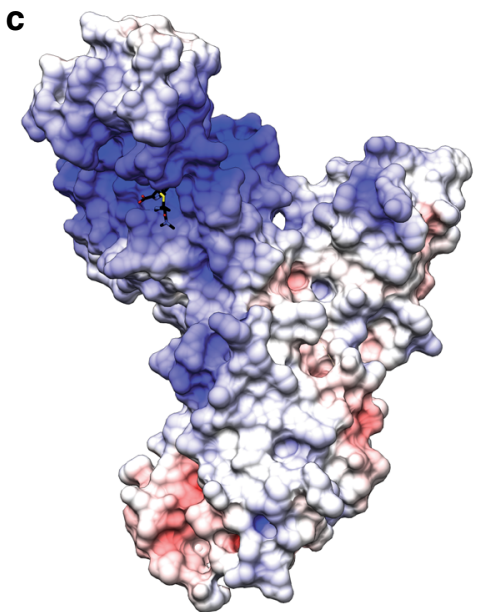

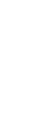
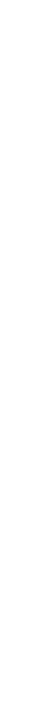

d Gm -ve Capsule Mtb Gm +ve

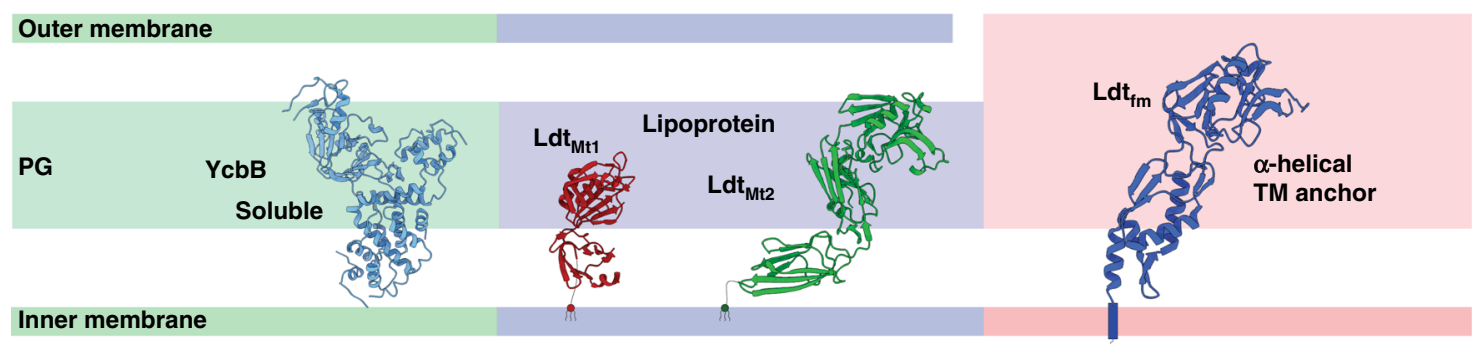

Fig. 1 Overall architecture of $E$. coli YcbB. a The crystal structure of YcbB-meropenem acyl-enzyme complex in ribbon representation, coloured in rainbow from $\mathrm{N}$-terminus (blue) to C-terminus (red). Meropenem stick representation is coloured in black and by heteroatom. Two views related by a $90^{\circ}$ rotation along the $y$-axis. b Topology diagram of YcbB-meropenem acyl-enzyme complex, coloured as in $\mathbf{a}$. The catalytic, PG and scaffolding domains are annotated and circled in grey. c Electrostatic surface representation of $Y c b B$ in two views related by a $180^{\circ}$ rotation around the $y$-axis. $\mathbf{d}$ Diversity of structurally characterised L,D-transpeptidases. Gram negative (green background), soluble YcbB in sky blue; Mycobacterial (blue background), lipoprotein Ldt ${ }_{\text {Mt1 }}$ (PDBID 5E5L ${ }^{8}$ ) and $\mathrm{Ldt}_{\mathrm{Mt2}}$ (PDBID 5DU78) in red and green; Gram positive (red background), TM anchored $\mathrm{Ldt}_{\mathrm{fm}}\left(1 \mathrm{ZAT}{ }^{16}\right)$ in dark blue

mediated reistance ${ }^{5}$. Residues 234-351 form a small antiparallel three helical bundle, with features reminiscent of previously observed PG binding regions-and thus we term here the PG domain ${ }^{11}$. Residues 577-604 form a beta-hairpin linker between the catalytic domain and the C-terminal recursion.

YcbB scaffold domain structure. The scaffold domain of YcbB is comprised of ten helices packed into a helical bundle. The seven core $\alpha$-helices are contributed by residues $100-213$, with the eighth $a$-helix contributed from the C-terminal recursion involving residues $600-615$ (Fig. 1). Two additional helices (residues 214-233 and 352-374) cap the domain and structurally link the catalytic and PG binding domains. Residues downstream (31-99) form a generally less ordered region with interspersed $\alpha$-helical segments which sit distal to the other domains of YcbB. The scaffold domain is unique to the YcbB sub-family of L,D-transpeptidases, suggesting a specific functional and/or regulatory role 
a

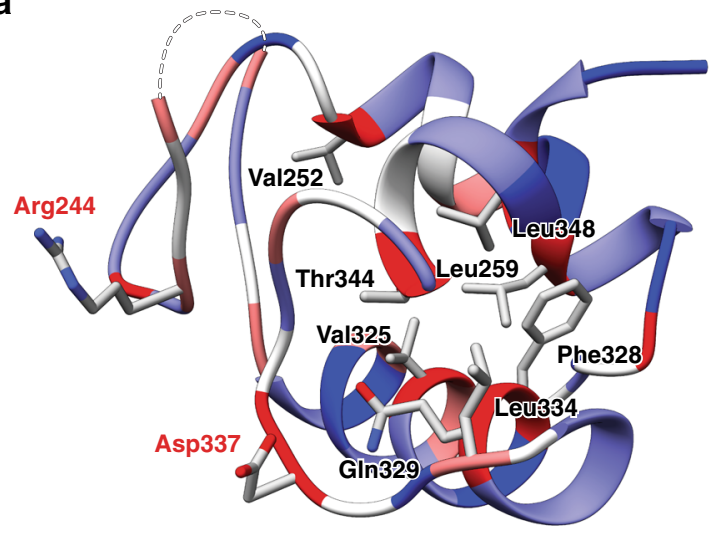

b

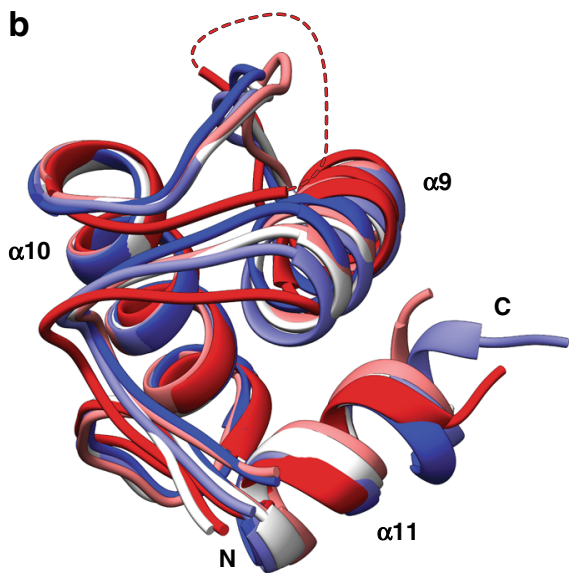

c

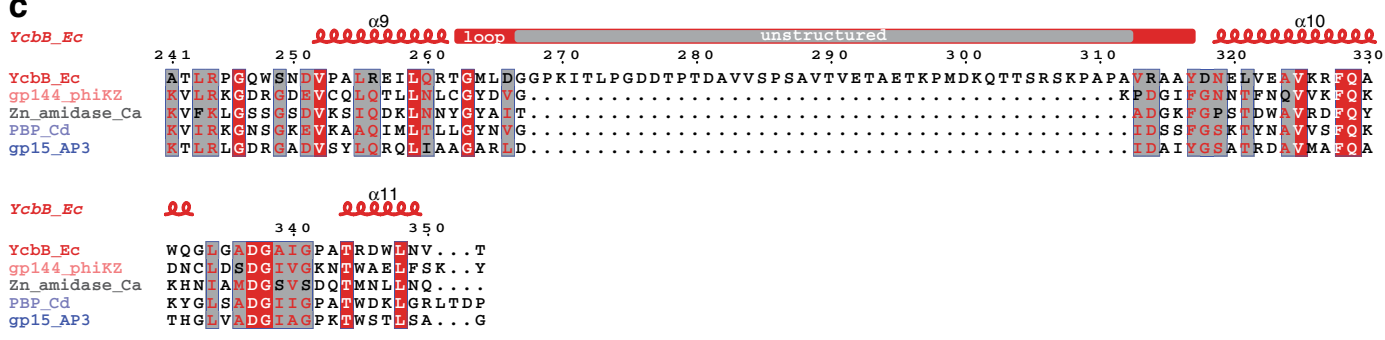

Fig. 2 Structure of the E. coli YcbB peptidoglycan binding domain. a Ribbon representation of the E. coli YcbB peptidoglycan binding domain, residues 241-351, with residue conservation mapped to backbone colour from red to blue in decreasing conservation. Conserved core residues labelled in black and key, surface exposed, peptidoglycan binding residues labelled in red. b Overlay of YcbB peptidoglycan binding domain and high scoring peptidoglycan binding domains from a structural homology search on the Dali server ${ }^{14}$. YcbB in red, gp144, a Pseudomonas aeruginosa phage phiKZ endolysin in salmon (PDB ID 3BKH ${ }^{15}$ ), a Clostridium acetobutylicum zinc-dependent amidase in white (PDB ID 4XXT), a Clostridium difficile PBP in pale blue (PDB ID 5TV7), and gp15, a Burkholderia AP3 phage endolysin in dark blue (PDB ID 5NM711). Peptidoglycan binding domain structures are coloured from red to blue based on decreasing structural homology. c The sequence of E. coli YcbB (YcbB_Ec) aligned with peptidoglycan binding domains from phiKZ gp144 (gp144_phiKZ), C. acetobutylicum zinc-dependent amidase (Zn_amidase_Ca), C. difficile PBP (PBP_Cd), and AP3 gp15 (gp15_AP3). Secondary structure of E. coli YcbB is displayed atop the sequence alignment, with the extended loop shown in red and the unstructured region in grey

we predict to involve interaction with the known YcbB unique protein partners $\mathrm{PBP} 5$ and $\mathrm{PBP} 1 \mathrm{~b}^{5}$. These interactions are likely crucial for localization of $\mathrm{YcbB}$ to the site of late stage PG synthesis, as, unlike L,D-transpeptidases from Gram positive bacteria or Mycobacterium, YcbB does not have a a-helical transmembrane tether or N-terminal lipidation (Fig. 1d). Rather, $\mathrm{YcbB}$ is predicted to be targeted to the periplasmic space using a characteristic sec-dependent signalling sequence (residues 1-30SignalP $4.1^{12}$ ), which is subsequently processed through proteolytic cleavage with release of soluble YcbB.

YcbB peptidoglycan binding domain. Many characterized L,Dtranspeptidases have been shown to contain PG binding motifs/ domains, often a LysM domain consisting of a $\beta \alpha \alpha \beta$ fold with the two $\alpha$-helices located on the same side of an antiparallel betasheet ${ }^{13}$. In YcbB, residues 234-351 form a small antiparallel three helical bundle which, based on these prior observations, is suggestive of a putative PG binding domain (Fig. 2) albeit a PG binding domain more characteristic of lytic phage enzymes and a Zn-dependent bacterial amidase than PG binding motifs observed in other structurally characterized L,D-transpeptidases. Structural homology analysis, via the DALI protein structure comparison server ${ }^{14}$, finds close structural homologues-gp144, a Pseudomonas aeruginosa phage phiKZ endolysin (PDB ID $3 \mathrm{BKH}^{15}, \mathrm{Z}$ score 9.7, RMSD 1.9), a Clostridium acetobutylicum zincdependent amidase (PDB ID 4XXT, Z-score 9.6, RMSD 1.9), a Clostridioides difficile PBP (PDB ID 5TV7, Z-score 9.4, RMSD 2.0), and gp15, a Burkholderia AP3 phage endolysin (PDB ID $5 \mathrm{NM} 7^{11}$, Z-score 9.4, RMSD 2.0). The three antiparallel helices are common to all five PG binding domains, though in YcbB the loop region between the first and second helices contains a dynamic and relatively conserved extension (residues 262-318 with the central region therein highly disordered). Due to the lack of this loop extension in the above identified structural homologues, it is possible that these residues become more stabilised upon PG binding, increasing the effective interaction interface or specificity with PG substrates in this family. The PG domain of YcbB contains many conserved residues with other YcbB family members primarily in the hydrophobic core of the motif (Val252, Leu259, Ala324, Val325, Phe328, Gln329, Leu334, Thr344, and Leu348) as well as in two surface exposed residues, Arg244 and Asp337, which have been implicated previously as essential in PG binding ${ }^{11}$.

YcbB catalytic domain. The central YcbB catalytic domain adopts a characteristic L,D-transpeptidase fold (Fig. 3) constructed of two (five and six stranded) curved, mixed beta-sheets disposed in a clam-shell like manner with $\alpha$-helices 15 and 16 situated in the cleft between. Acting as a hinge, beta-strand 3 participates in both beta-sheets of the domain. Situated just downstream of beta-strand 11, the active site is demarcated by the meropenem acylated catalytic cysteine nucleophile (Cys528) with the conserved histidine base (His509) protruding from betastrand 10 . The oxyanion hole, presumed to stabilize the developing tetrahedral oxyanion transition state during acylation and deacylation is comprised of the main chain nitrogen atoms of Cys528 and Tyr507. 


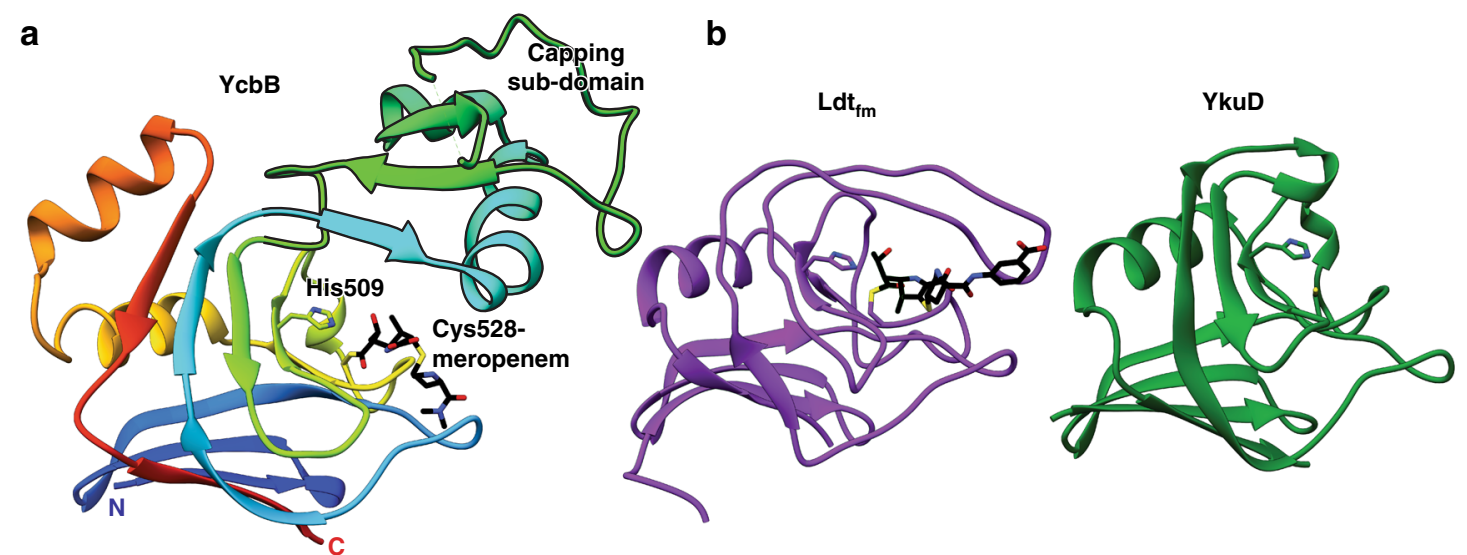

C

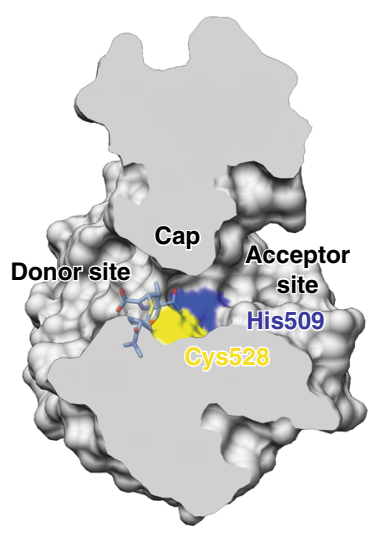

d

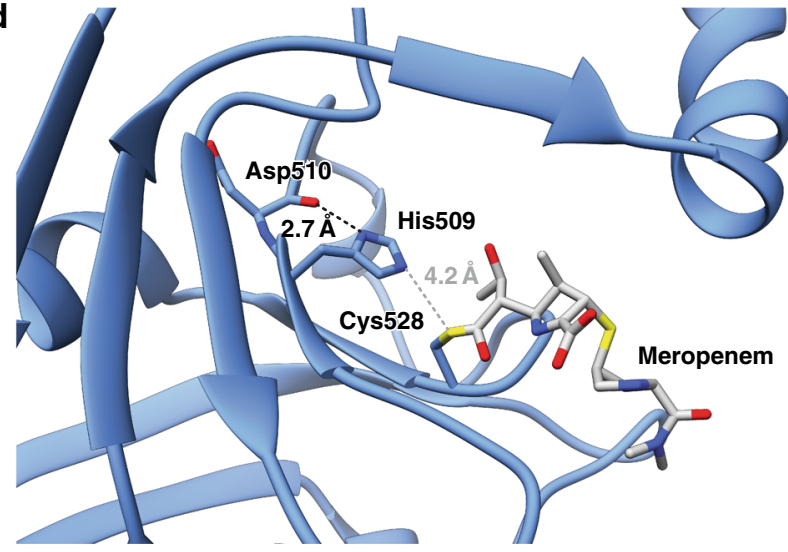

e
EC_YCbB
EC_YcbB
Bs_YkuD
Ef_LdtFm
Mt_LdtMt1
Mt_LdtMt2
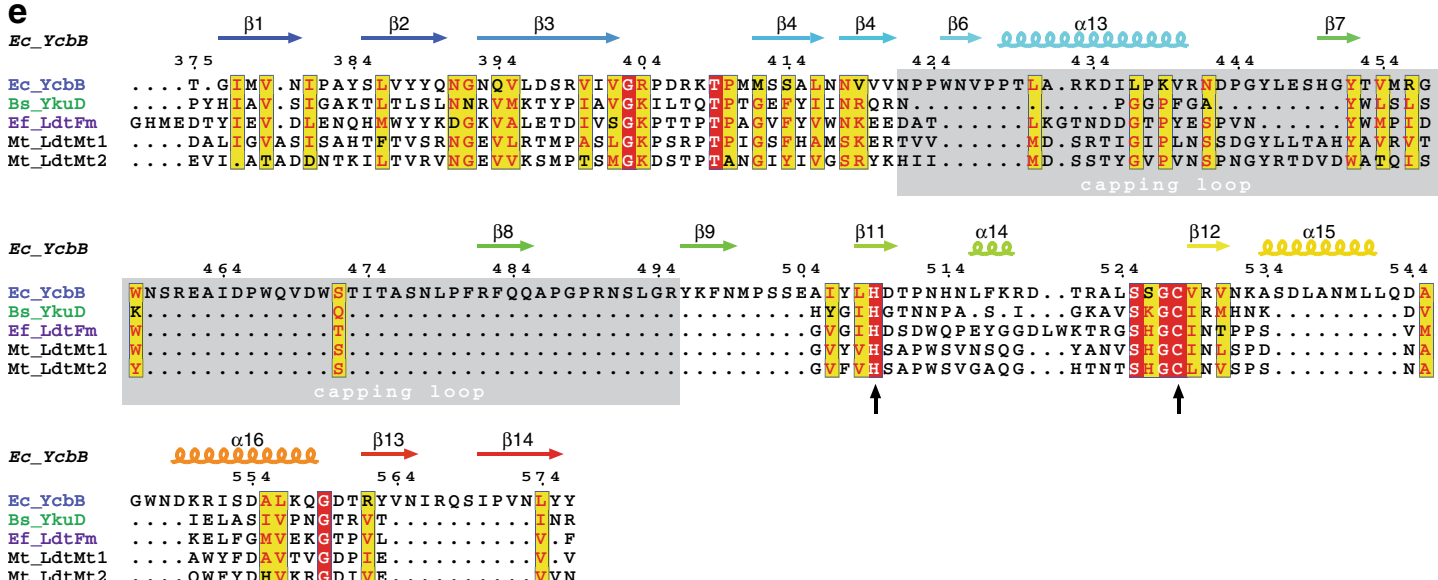

Fig. 3 Structure of the E. coli YcbB catalytic domain. a A ribbon representation of the YcbB catalytic domain, residues 375-576, coloured in rainbow from red ( $\mathrm{N}$-terminus) to blue (C-terminus). Meropenem is represented in black and coloured by heteroatom. The unique capping sub-domain is highlighted in a bold silhouette. $\mathbf{b}$ The catalytic domain architecture of Enterococcus faecium Ldt $_{\mathrm{Fm}}$ (PDB ID 3ZGP17) and Bacillus subtilis YkuD (PDB ID 1Y7M ${ }^{13}$ ) highlighting the differences seen among L,D-transpeptidase catalytic domains in the capping loop region. c Cut surface representation of the YcbB catalytic domain, showing the capped cleft formed between the donor and acceptor sites by the capping loop sub-domain. The catalytic Cys528 and His509 are coloured in yellow and blue respectively. Meropenem (blue) is overlaid in its position in the donor site, acylating the catalytic cysteine. $\mathbf{d}$ The active site architecture of E. coli YcbB. The catalytic Cys528 is acylated by meropenem and the sulphide is rotated away from the $\mathrm{N}^{\tau}$ of His509 (4.2 $\AA$ ) which activated the cystine prior to acylation. The $\mathrm{N}^{\pi}$ of His509 is seen to be in close proximity with the backbone carbonyl of the adjacent Asp510 (2.7 $\AA$ ), stabilising and promoting formation of the histidine cation. e The sequence of E. coli Y $c b B\left(E c_{-} Y c b B\right.$ ) aligned with the aforementioned catalytic domains from E. faecium and B. subtilis (Ef_Ldtfm and Bs_Yku, respectively) as well as $\mathrm{Ldt}_{\mathrm{Mt} 1}$ and $\mathrm{Ldt}_{\mathrm{Mt2}}$ from Mycobacterium tuberculosis (Mt_LdtMt1 and Mt_LdtMt2, respectively)

One notable feature of the catalytic domain of YcbB is the large substrate capping sub-domain (residues inserted between betastrands 5 and 9). This region has been seen to be variant in currently solved structures of L,D-transpeptidases, ranging from small turns $\left(\mathrm{YkuD}^{13}\right)$ to larger loops of up to 19 residues which extend over the active site $\left(\mathrm{Ldt}_{\mathrm{fm}}{ }^{16,17}\right)$ (Fig. 3b). However, $\mathrm{YcbB}$ is the first structure characterized which contains significant secondary structural elements and consequent folded subdomain in this region (residues 423-487). In YcbB this putative substrate capping sub-domain is comprised of a small three stranded beta-sheet $(\beta 6,8,7)$ and $\alpha$-helix 13 , which sits perpendicular to the beta-sheet at one end. Between beta-strand 7 and 8 
there is a large loop region from residue 453 to 481 . Beta-strand 6 and $\alpha$-helix 13 are seen to hinge towards the active site in the meropenem acyl-enzyme complex structure. It has been proposed previously that the substrate capping loop in earlier structures serves to provide inherent flexibility during roles in substrate entrance, capping, and release ${ }^{18}$. Despite the more structured and significant nature of the YcbB sub-domain, the density, although traceable, was less well resolved and displayed generally higher temperature factors, all in keeping with an analogous inherent flexible nature and potential similar role(s) in catalysis. Further, crystallographic structures and molecular dynamics simulations of $\mathrm{Ldt}_{\mathrm{Mt} 2}$ from Mycobacterium tuberculosis have recently shown that the substrate capping loop can have variable flexibility dependent on the presence of differing inhibitors with obvious implications for drug discovery efforts against this family ${ }^{18,19}$. To probe further the potential analogy with the more structured sub-domain in YcbB, we performed $10 \mathrm{~ns}$ molecular dynamics simulations (Supplementary Fig. 3) using a CHARMM36 force field (see Methods) which additionally supported the propensity for its highly mobile nature. Interestingly, simulations with meropenem showed increased RMSD spread in the capping loop region but an overall stabilisation of the remainder of the catalytic domain, while in apoenzyme simulations there was increased motion not only in the capping sub-domain but as well over the entirety of the catalytic domain. It is likely that in the action of YcbB on extended native PG substrates, this motion would provide for additional interactions to facilitate binding and product release. In the YcbB-meropenem acyl-enzyme complex structure, the capping subdomain is seen to be stabilised in a position adjacent to the active site, creating a distinct cap over the two substrate binding clefts (Fig. 3c). Meropenem occupies, as expected of a beta-lactam antibiotic, the donor site, further demarcating the adjacent and unoccupied acceptor site. The corresponding volume under the cap in the closed conformation observed is $\sim 810 \AA^{3}$, as calculated by the $3 \mathrm{~V} \operatorname{server}^{20}$ (Supplementary Fig. 4).

YcbB L,D-transpeptidase mechanism. From the structure of meropenem acylated $\mathrm{YcbB}$, we propose a catalytic mechanism of the L,D-transpeptidase activity of $\mathrm{YcbB}$, which despite the architectural differences is in keeping to what has been proposed for other L,D-transpeptidases previously ${ }^{21}$ (Supplementary Fig. 5). In the proposed mechanism, the conserved Cys528 carries out a nucleophilic attack on the penultimate residue, meso-DAP, of a tetrapeptide on the donor PG strand, resulting in the release of the terminal D-Ala. This acyl-enzyme intermediate is subsequently deacylated via nucleophilic attack of a side chain amino on the meso-DAP on an adjacent acceptor PG strand. Conserved His509 of the catalytic dyad is seen to be located in close proximity to the catalytic Cys528 (distance $\mathrm{N} \tau$ to acylated Cys $\mathrm{S}$ of $4.2 \AA$ ); we suggest the hydrogen bond distance and angle between base and nucleophile are presumably optimized only in the apo form of the enzyme due to steric restraints induced by the covalently bound substrate in the subsequent acyl intermediate as captured here. Indeed several structures of acylated and apo forms of PBPs support this with high resolution structures showing the hydroxyl group of the catalytic serine nucleophile swings away from the conjugate base upon acylation ${ }^{22}$. As well, we observe this in our apo molecular dynamics simulations, where the cysteine swings toward His509 within $10 \mathrm{~ns}$ of simulation to a final distance of $3.1 \AA$ between the His $\mathrm{N} \tau$ to apo Cys $\mathrm{S}$. The backbone carbonyl of the adjacent Asp510 is seen to be located near the $\mathrm{N} \pi$ of His509 (2.7 $\AA$ ), forming a stabilising hydrogen bond and potentially modulating the $\mathrm{pK}_{\mathrm{a}}$ of the His509 to improve its role as a general base. His509 is seen to be positioned both proximal to not only the catalytic cysteine, but as well the acceptor site of the catalytic cleft (Fig. 3c, d). This supports the notion that His509, stabilised by Asp510, acts as the general base for the activation of both the acylation and deacylation steps of the L,D-transpeptidase reaction. It has been proposed previously for other L,D-transpeptidases that the catalytic histidine may act as both the general base, as well as to protonate the leaving groups ${ }^{21}$, but from our YcbB-meropenem complex structure we propose that Tyr507 is a more likely candidate for this protonation in the case of YcbB. Tyr507 is more appropriately positioned (Supplementary Fig. 1C), in angle and at $4.2 \AA$ from the leaving group nitrogen, than the catalytic histidine, which is positioned $7.2 \AA$ away and appears unlikely to donate this proton. As well, the nearby electropositive Lys497 (4.5 away) and potential hydrogen bond partner Trp 425 (3.6 A away) could serve to reduce the $\mathrm{pK}_{\mathrm{a}}$ of the Tyr507 and allow for its role in protonation of the leaving group. The role of the Tyr507 at this position would be analogous to the role of the SXN motif serine (Ser139 in PBP5) in PBPs $^{23}$, a role which has been very well characterized. We note that while a tyrosine residue exists at this position in L,D-transpeptidases from a variety of other species, it is not completely conserved at this position in the L,D-transpeptidase motif. The specific nature of these protonation events can perhaps be unravelled in future Michaelis complex structures of L,D-transpeptidases.

Inhibition of the L,D-transpeptidase mediated resistance pathway. In order to further probe the inhibition of the YcbBmediated beta-lactam resistance pathway, meropenem acylation of both the L,D-transpeptidase and D,D-carboxypeptidase activities required was investigated (Fig. 4). The YcbB-meropenem complex map had ordered density defining the active site residues including the thiol ester covalent link of Cys528 and acylated meropenem (Fig. 4b). A $2.2 \AA$ resolution structure of PBP5 acylated with meropenem was also solved with very well ordered density for the covalently bound meropenem (Fig. 4c). Relative to previously published PBP5 structures, we were able to trace additional residues at the C-terminus of PBP5 (387-397; likely afforded by the differential packing with a neighbouring molecule in our unique crystal lattice). Regardless, the conserved binding mode of the ligands between the PBP5-meropenem structure and the PBP5-imipenem structure (PDB ID 3MZF ${ }^{24}$ ) indicates similar modes of enzyme acylation and binding. On the other hand, despite the similarity in the PG substrates for the YcbB L,Dtranspeptidase and PBP5 D,D-carboxypeptidase, we observe the binding and stabilisation of meropenem between the two enzymes is quite distinct.

Meropenem binds PBP5 in a typical PBP fashion using the conserved SXXK, SXN, and KTG active site sequence motifs common to this broad class of activated serine carboxypeptidases. The meropenem is acylated at C7 by the Ser73 side chain hydroxyl of the SXXK motif (the proximal Lys76 of the motif playing the general base role) and stabilised through an extensive hydrogen bond and electrostatic network (Fig. 4c, g). The carbonyl oxygen on C7 of meropenem occupies the enzyme oxyanion hole comprised of main chain nitrogens at position 73 and 245, forming strong hydrogen bonds that polarize and stabilize the carbonyl during acylation and formation of oxycarbanion intermediates (Fig. 4d). The side chain hydroxyl of Ser 139 of the SXN motif resides adjacent to the leaving group nitrogen of meropenem, the likely source for protonation of that moiety to promote acylation. The Asn141 side chain amide of the SXN motif hydrogen bonds with the 1-hydroxyethyl group on C6 of meropenem, while the Thr243 side chain hydroxyl of the KTG motif forms bidentate hydrogen bonds to the carboxylic acid 
<smiles>CC(O)C1C(=O)N2C(C(=O)O)=C(SC3CNC(C(=O)N(C)C)C3)C(C)C12</smiles>

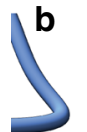

$\int^{6}$
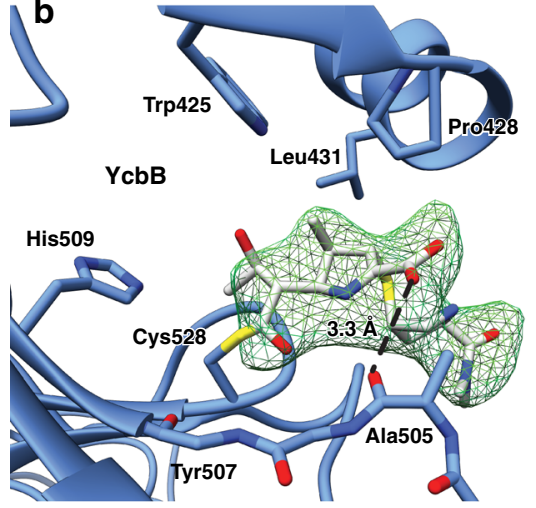

f

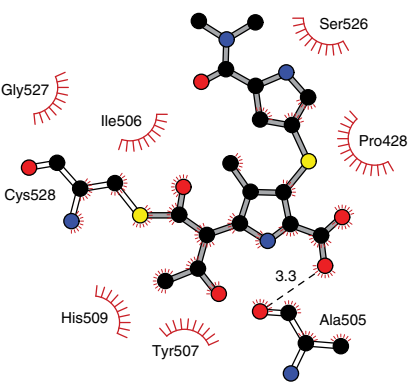

g

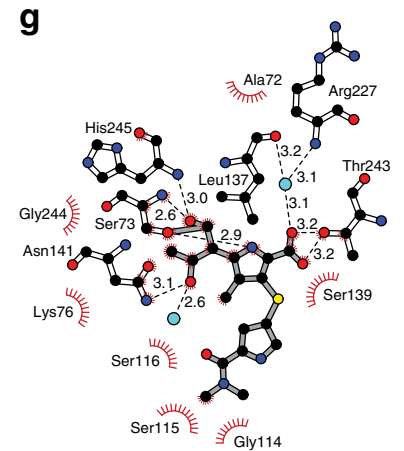

d

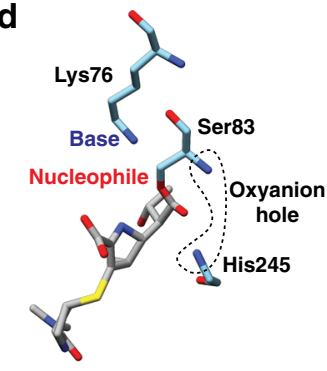

$\mathbf{e}$

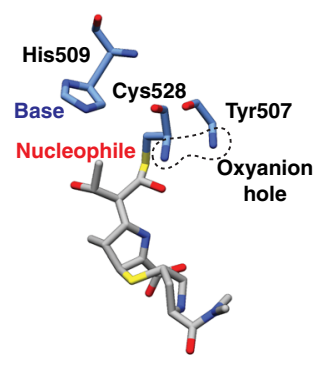

Fig. 4 Meropenem inhibition of E. coli YcbB and PBP5. a Chemical diagram of meropenem pre- and post-acylation by $\mathrm{YcbB}_{\mathrm{c}}$ and $\mathrm{PBP}_{\mathrm{B}}$. $\mathbf{b} \mathrm{mF} \mathrm{F}_{\mathrm{o}}-\mathrm{DF} \mathrm{F}_{\mathrm{c}}$ simulated annealing omit map for the acylated meropenem on Cys528 of $Y c b B$, contoured at $2 \sigma$. YcbB is in blue and meropenem in grey. The catalytic dyad (Cys528 and His509), selected residues, and meropenem are coloured by heteroatom. Hydrogen bonding between meropenem and the backbone carbonyl of Ala505 is represented with dashed lines. $\mathbf{c} \mathrm{mF}_{\mathrm{o}}-\mathrm{DF}_{\mathrm{c}}$ simulated annealing omit map for the acylated meropenem on Ser73 of PBP5, contoured at $2 \sigma$. PBP5 is in blue and meropenem in grey. PBP5 residues involved in the stabilization of meropenem, relevant water molecules, and meropenem are coloured by heteroatom. Hydrogen bonding between meropenem, selected residues, and water is represented with dashed lines. Relative arrangement of nucleophile, general base and oxyanion hole (backbone atoms shown) for PBP5 (d) and YcbB (e). f, $\mathbf{g}$ LigPlot diagrams of meropenem acyl-enzyme complexes. $\mathbf{f}$ YcbBmeropenem complex with meropenem in grey and protein residues in white, with atoms coloured by heteroatom. $\mathbf{g}$ PBP5-meropenem complex coloured as in $\mathbf{f}$. $\mathbf{h}$ Overlay of meropenem-acylated catalytic residues of YcbB (darker blue and green) and PBP5 (lighter blue and pink) showing a similar general curvature of both meropenem molecules, with the notable exception of an approximate $180^{\circ}$ rotation about meropenem $\mathrm{C} 6$ due to the differing active site architectures of $\mathrm{YcbB}$ and PBP5

group of meropenem (C3). The latter is further stabilized by longer range electrostatic interactions with the side chain amido of Lys242 of the motif as well as the guanidinium group of Arg277. As well there are typical hydrogen bonds formed, backbone amide of His 245 and water-mediated interactions with the backbone amide of Arg227 and the backbone carbonyl of Leu137.

In contrast, meropenem binding of $\mathrm{YcbB}$ is void of this extensive network of hydrogen bonds, instead consisting of acylation at $\mathrm{C} 7$ by the catalytic Cys528 and a few key hydrogen bonds (Fig. 4b, f). The carbonyl oxygen on C7 of acylated meropenem protrudes into an oxyanion hole consisting of, analogous to the PBPs, the amide nitrogen of the nucleophile, here Cy528. However, the second commonly observed main chain nitrogen amide of the PBPs is not present in YcbB, instead a close interaction to the carbonyl oxygen at Ala 505 with substrate is observed. A further markedly distinct feature in the meropenem complex is the minimal non-covalent interactions of the C3 carboxylate of meropenem with enzyme. One hydrogen bond to the backbone carbonyl of Ala505 is the primary interaction, replacing the typical electrostatic interactions observed for this ubiquitous functional group of beta-lactam antibiotics. Despite the relative proximity of electropositive groups such as Arg407, Arg433, Lys434, and Lys497, the proline rich insertion centered at Pro428 appears to provide a structural ridge that prevents significant interaction. This ridge perhaps contributes to the molecular basis for generally poor acylation of YcbB by classic beta-lactam antibiotics compared to classical PBPs, where the electrostatic positioning of the substrate carboxylate by conserved electropositive side chains is a critical, reoccurring theme. At the same time hydrophobic interactions between enzyme and substrate appear more prominent, with, for example, Trp425, Pro428 and Leu431 forming an explicit apolar pocket for binding of the $\mathrm{C} 1$ methyl of meropenem. The general 
lack of complex hydrogen bonding and increased hydrophobic interactions is characteristic of L,D-transpeptidase acyl-enzyme complexes with carbapenem inhibitors and is seen in meropenem acylation of $\mathrm{Ldt}_{\mathrm{Mt} 2}$ (PDB ID 4QR7 ${ }^{18}$ ). Pre-acylation, there could be additional stabilisation of meropenem from residues of both the active site and the capping loop that allow for proper positioning of the drug for acylation by the catalytic cysteine.

Despite the similarities in the activities of PBP5 and YcbB and therefore some of the common attributes to the inhibition of these enzymes by meropenem, there are major differences in the overall stabilization of these drugs post-acylation. It is likely that PBPs were the target of the evolution of this class of antibiotics, originally in Streptomyces cattleya and in subsequent drug development ${ }^{25}$. This is exemplified by structural properties of the inhibition of these enzymes, with the generally more stabilized meropenem of the acylated PBP5 proving a better inhibitor. As we continue to unravel the importance of L,D-transpeptidase mediated beta-lactam resistance, it will become increasingly relevant to revisit this important class of antibiotic and, using structure guided design, improve upon the binding and inhibition of L,D-transpeptidases by carbapenems.

In vivo assay of $\mathrm{YcbB}$ mediated beta-lactam resistance. In order to correlate the sequence and structural aspects of E. coli $\mathrm{YcbB}$ to its role in antibiotic resistance, antibiograms using a beta-lactam disk diffusion assay were performed (Fig. 5). The antibiotics
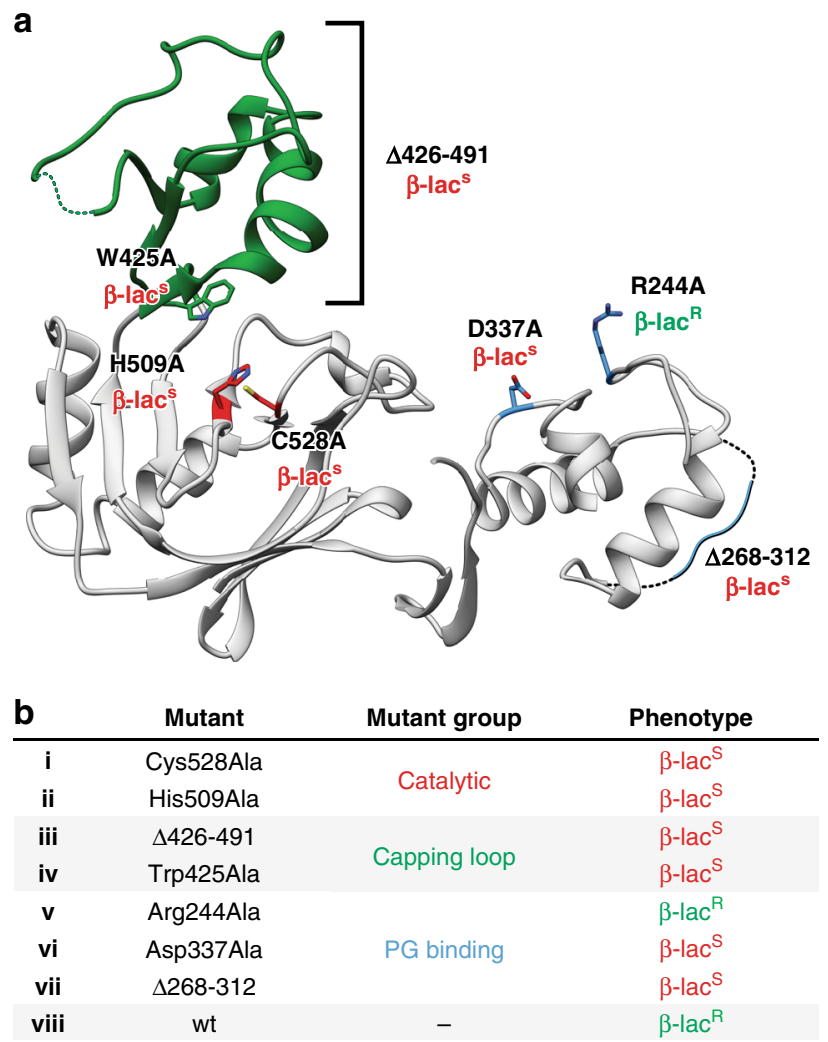

Fig. 5 In vivo assay of $Y c b B$ mediated beta-lactam resistance. a Various replacements and deletions of the catalytic residues (in red), capping loop region (in green) and $P G$ binding domain (in blue), mapped onto the catalytic and PG domains of YcbB. Replacements retaining YcbB mediated beta-lactam (ampicillin and ceftriaxone) resistance are denoted as $\beta-l a c^{R}$, while replacements and deletions resulting in beta-lactam (ampicillin and ceftriaxone) susceptibility are denoted as $\beta-\mathrm{lac}^{S}$. b Replacements and deletions of $\mathrm{pTK} 2(y c b B)$ (i-viii) are listed with their resulting phenotype ampicillin and ceftriaxone were chosen for use in these assays, as they are well characterized, non-carbapenem, beta-lactam antibiotics that provide insight into the general trends in beta-lactam resistance apart from carbapenems. Unsurprisingly, replacements of both the catalytic cysteine and histidine resulted in a complete loss of the beta-lactam resistance $\left(\beta-l a c^{R}\right)$ phenotype seen in the assay with wild type YcbB.

Of greater interest was the contribution of the capping loop to antibiotic resistance. To this end, the assay was performed with a truncated YcbB containing a YkuD-like capping stub $(y c b B$ $\Delta 426-491)$, as well as with a Trp425 Ala replacement designed to interfere with the significant hydrophobic interactions the indole side chain was observed to mediate with meropenem in our complex structure. Both of these replacements resulted in an beta-lactam sensitivity $\left(\beta-\operatorname{lac} c^{S}\right)$ phenotype. This further validates the proposition that the capping loop, despite its inherent flexibility, plays a crucial role in facilitating YcbB mediated L,Dtranspeptidation.

Replacements of the catalytic domain and capping sub-domain provide insight into the requirement for functional L,Dtranspeptidase activity in antibacterial resistance, yet an understanding of the role of the PG domain in this resistance and the activity of L,D-transpeptidases in general was poorly understood. While many L,D-transpeptidase enzymes have PG domains of one type or another, these domains have yet to be specifically linked to their role in LD-transpeptidation in vivo. Replacements of both the presumed PG binding residues of the YcbB PG domain (Asp337 and Arg244), as well as a truncation to the extended loop (ycbB $\Delta 268-312$ ) unique to the YcbB PG domain (in comparison to homologous PG domains in other enzymes), were tested using the in vivo beta-lactam resistance assay. Interestingly, only the Asp337Ala replacement and $\Delta 268-312$ deletion resulted in beta-lactam sensitivity. The Arg244Ala replacement resulted in a continued beta-lactam resistance phenotype. We propose that this is likely due to a greater contributing role of Asp337 in the binding of PG in comparison to Arg244. Asp337 is positioned more closely to the cleft between the catalytic and PG domains (Fig. 5a), where the acceptor PG strand would likely lie in order to have access to the acceptor site of the catalytic domain. In contrast, Arg244 is positioned further away from the cleft and less likely to be forming as strong an interaction with the acceptor PG strand (Fig. 5a). Regardless of the contribution of Arg244 in PG binding, this is the first evidence of how an accessory PG binding domain can have implications on L,D-transpeptidase function in an in vivo setting. As the extended loop of the PG domain is on the opposite face from residues proposed to be involved in binding the acceptor PG strand, it is possibly mediating additional interactions with the existing $P G$ sacculus that are important in the context of the greater YcbB mediated resistance pathway. Overall, this clearly shows the importance of the accessory PG binding domains in L,D-transpeptidase function in vivo.

Interaction of YcbB with PBP1b and PBP5. Ycbb, PBP5 and $\mathrm{PBP} 1 \mathrm{~b}$ are known to contribute sequentially in the D,D-transpeptidase bypass pathway in $E$. coli $i^{5}$. It was of interest, therefore, to determine whether YcbB interacts directly with these PBPs that provide substrate. To do this assessment, YcbB-PBP1b and YcbB-PBP5 interactions were assayed using microscale thermophoresis (MST). Styrene-maleic acid co-polymer solubilised PBP1b was used to mitigate protein aggregation issues in the capillary that were encountered with detergent-solubilised PBP1b. The YcbB-PBP1b interaction was seen to have a $K_{\mathrm{d}}$ of $250 \pm 30 \mathrm{nM}$ (Supplementary Fig. 6A). The YcbB-PBP5 interaction was seen to be somewhat weaker, with the MST data 


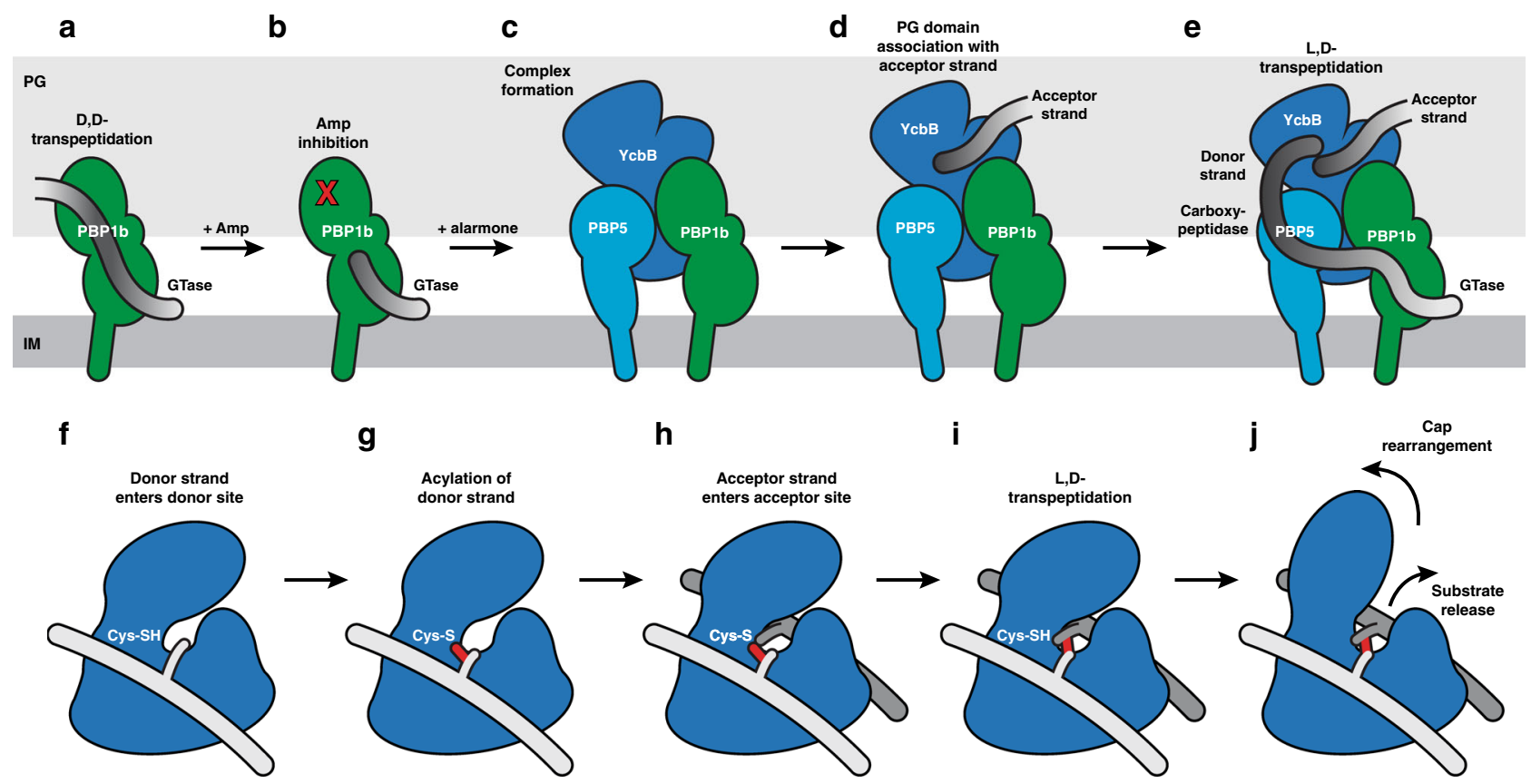

Fig. 6 Schematic representation of YcbB mediated beta-lactam resistance. a-e Assembly and function of the YcbB mediated beta-lactam resistance pathway. a PBP1b function in the absence of beta-lactam antibiotics. PBP1b acts as both glycosyltransferase and D,D-transpeptidase. $\mathbf{b}$ PBP1b function in the presence of beta-lactam antibiotics, such as ampicillin. PBP1b can maintain glycosyltransferase activity, but D,D-transpeptidation is inhibited. c Betalactam resistance complex formation upon production of alarmone. YcbB likely interacts with PBP1b and PBP5 through its putative interaction/helical domain. $\mathbf{d}$ YcbB is likely additionally associated and oriented through its PG binding domain interacting with the acceptor strand of the existing PG sacculus. e The resistance complex can successfully rescue crosslinking function. PBP1b polymerizes PG strands with glycosyltransferase activity, PBP5 removed a terminal D-alanine with its carboxypeptidase activity, and $Y c b B$ acts on the modified peptides with L,D-transpeptidase activity to crosslink the PG strand into the sacculus. f-j Function and mechanism of the YcbB catalytic domain during L,D-transpeptidation. $\mathbf{f}$ Tetrapeptide of the donor PG strand enters the donor site of the catalytic domain. $\mathbf{g}$ Donor tetrapeptide is acylated at its meso-DAP residue by the catalytic cysteine, resulting in the loss of the terminal Dalanine. $\mathbf{h}$ Adjacent acceptor PG peptide enters the acceptor site of the catalytic domain. $\mathbf{i}$ The covalent acyl-enzyme is deacylated via nucleophilic attack of the side chain meso-DAP on the adjacent acceptor PG strand, forming the L,D-crosslink. $\mathbf{j}$ Rearrangement of the capping loop/sub-domain from a capped conformation to an open cleft will occur, resulting in the release of the crosslinked substrate

suggesting at most a $K_{\mathrm{d}}$ in the low micromolar range $(\sim 2 \mu \mathrm{M})$ (Supplementary Fig. 6B). These affinities are consistent with the generally-accepted understanding regarding the dynamic nature of PG biosynthetic complexes in response to environmental challenges or the particular stage in the life cycle of the bacterium $^{26}$. Due to the relatively apolar nature of the scaffolding domain of YcbB (Fig. 1) we postulate that these interactions with PBP1b and PBP5 primarily occur through interactions with apolar regions of PBP1b and PBP5. Both PBP1b and PBP5 are seen to exhibit similar electrostatic surface charge characteristics to $\mathrm{YcbB}$, with localized electropositive surface regions about their active sites and more apolarity to their other surface regions (Supplementary Fig. 7). The association of YcbB to the apolar hinge region of PBP5 and the apolar regions of PBP1b's glycosyltransferase domain would localise YcbB to receive the proper substrates from PBP1b and PBP5 to successfully mediate betalactam resistance.

Implications on YcbB mediated beta-lactam resistance. The structures reported here further develop our understanding of YcbB mediated beta-lactam resistance and allow us to expand upon the previously proposed model of the resistance pathway ${ }^{5}$ (Fig. 6). Under conditions of increased alarmone production, YcbB and PBP5 are likely able to form a transient complex with PBP1b. The soluble protein YcbB is likely able to couple to the two monotopic membrane proteins, PBP1b and PBP5, to interact directly through its putative interaction/helical domain. As well, YcbB is likely assisted in this localisation and oriented in position to crosslink a polymerising PG strand by its PG binding domain mediated interaction with the existing sacculus. This is distinct from the role of the LysM-like PG binding domain of $\mathrm{YkuD}^{27}$ and other Ldt enzymes, in which the PG binding domain is oriented adjacent to the donor site of the catalytic domain, as opposed to the acceptor site seen in YcbB (Supplementary Fig. 8). Once the complex has been formed, PBP1b could act to polymerise periplasmicaly oriented lipid II into a growing PG strand. As betalactam drugs inhibit the transpeptidase domain of PBP1b, PBP5 could then assist in rescue of PG formation by removing the terminal D-Alanine, thereby providing the substrate for YcbB. This growing strand would orient itself adjacent to the donor site of YcbB, with the tetrapeptide stem entering into the catalytic cleft. In parallel, the PG domain associated acceptor PG strand would enter the acceptor site of the catalytic cleft. YcbB mediated L,D-transpeptidase activity and substrate release would occur involving rearrangement of the substrate capping loop/subdomain and resulting in the capped substrate cleft (seen in the acyl-enzyme complex structure) to re-orient into an open cleft. This proposed mechanism for this resistance pathway is consistent with the growing understanding in the field that PG biosynthesis depends on a number of dynamic and variable multiprotein complexes ${ }^{26}$ that are regulated spatiotemporally and, as in the case of YcbB mediated beta-lactam resistance, by external stimuli. As we enter into an era of increasing antibiotic resistance, unravelling the intricacies of these non-canonical PG biosynthetic complexes will be of increasing importance as bacteria deploy these flexible pathways to evade antibiotic assault. 


\section{Methods}

Cloning and protein expression. Escherichia coli YcbB without its signal peptide (residue 31 onward) was cloned into the expression vector pET41b-GST with a thrombin cleavable, C-terminal 8x His-tag. E. coli PBP1b from residue 58 onward was cloned into the expression vector pET41b-GST with a thrombin cleavable, Cterminal 8x His-tag. E. coli PBP5 from residue 30 onward was cloned into the expression vector pET41b-GST with a human rhinovirus (HRV) 3C protease cleavable, C-terminal 8x His-tag. Expression constructs were transformed into $E$. coli BL21 (DE3) for expression of YcbB and PBP5. PBP1b expression construct was transformed into E. coli C41. Primers for cloning are listed in Supplementary Table 2. Cells were cultured in ZYP-5052 autoinduction media for $4 \mathrm{~h}$ at $37^{\circ} \mathrm{C}$ followed by overnight protein expression at $25^{\circ} \mathrm{C}$. Cells were pelleted and stored at $-80^{\circ} \mathrm{C}$ until required.

Protein purification. For purification of YcbB and PBP5, cell pellets were resuspended in lysis buffer (20 mM Hepes, $\mathrm{pH} 8.0,300 \mathrm{mM} \mathrm{NaCl}, 10 \%$ glycerol) and lysed by processing twice with a homogenizer ( $15 \mathrm{kPa}$; Avestin). Cellular debris was pelleted by centrifugation at $125,000 \times g$ for $1 \mathrm{~h}$. The resultant supernatant was loaded onto a $1 / 5 \mathrm{~mL} \mathrm{Ni}^{2+}$-saturated HisTrap HP Sepharose cartridge (GE Lifesciences), washed with $75 \mathrm{mM}$ imidazole in Buffer A (20 mM Hepes, pH 8.0, $300 \mathrm{mM} \mathrm{NaCl}$ ), and the protein was eluted with $300 \mathrm{mM}$ imidazole in Buffer A. $1 \mathrm{U}$ of HRV $3 \mathrm{C}$ protease or thrombin was added per $\mathrm{mg}$ of protein to remove the $\mathrm{N}$-terminal His-tag overnight at $4{ }^{\circ} \mathrm{C}$. Samples were purified further by size exclusion chromatography (SEC) with a Superdex 200 column (GE Lifesciences) equilibrated in Buffer B (20 mM Hepes, pH 8.0, $150 \mathrm{mM} \mathrm{NaCl})$. Fractions containing pure PBP5 or YcbB were pooled and concentrated to 10 to $40 \mathrm{mg} / \mathrm{mL}$. Protein was frozen rapidly in liquid nitrogen and stored at $-80^{\circ} \mathrm{C}$ until required. For purification of PBP1b, cell pellets were resuspended in lysis buffer $(20 \mathrm{mM}$ Hepes, $\mathrm{pH} 8.0,300 \mathrm{mM} \mathrm{NaCl}, 10 \%$ glycerol) and lysed by processing twice with a homogenizer (15 kPa; Avestin). Cellular debris was pelleted by centrifugation at $10,000 \times g$ for $30 \mathrm{~min}$. The resultant supernatant was centrifuged at $125,000 \times g$ for $1 \mathrm{~h}$ to pellet membranes. The membranes were solubilised in Buffer A with $1 \%$ (w/v) N-dodecyl-D-maltopyranoside (DDM) overnight at $4{ }^{\circ} \mathrm{C}$ and loaded onto a $1 / 5 \mathrm{~mL} \mathrm{Ni}^{2+}$-saturated HisTrap HP Sepharose cartridge (GE Lifesciences), washed with $75 \mathrm{mM}$ imidazole in Buffer A with $0.016 \%$ DDM, and the protein was eluted with $300 \mathrm{mM}$ imidazole. One unit of thrombin was added per $\mathrm{mg}$ of protein to remove the $\mathrm{N}$-terminal His-tag overnight at $4{ }^{\circ} \mathrm{C}$. Samples were purified further by SEC with a Superdex 200 column (GE Lifesciences) equilibrated in Buffer B with $0.2 \%$ DM. Fractions containing pure PBP1b were pooled and concentrated to 10 to $40 \mathrm{mg} / \mathrm{mL}$. Protein was frozen rapidly in liquid nitrogen and stored at $-80{ }^{\circ} \mathrm{C}$ until required. SMA-solubilised PBP1b was prepared as above, with the extraction occurring in $2 \%$ activated SMA and subsequent buffers having no detergent present.

Microscale thermophoresis. MST assays were conducted using a Monolith NT.115Pico (NanoTemper). YcbB, PBP5 and PBP1b were fluorescently labelled using Alexa Fluor 647 NHS Ester (Thermo Fisher Scientific). To evaluate the binding of PBP1b to YcbB, increasing concentrations of unlabelled YcbB $(1.5 \mathrm{nM}-3.1 \mu \mathrm{M})$ were used to titrate fluorescently labelled SMA-solubilised PBP1b at a constant concentration $(100 \mathrm{nM})$. To evaluate the binding of PBP5 to $\mathrm{YcbB}$, increasing concentrations of unlabelled PBP5 (120 pM-98 $\mu \mathrm{M})$ were used to titrate fluorescently labelled $\mathrm{YcbB}$ at a constant concentration $(15 \mathrm{nM})$. Experiments were carried out in a Buffer C. Data was analysed using the NanoTemper MO Affinity Analysis software. The PBP1b-YcbB interaction data was analysed using fluorescence values, while the PBP5-YcbB data was analysed using MST. The data was fit to the equation for a binding isotherm with adjustable Hill coefficient.

X-ray crystallography and structure determination. E. coli YcbB protein was crystallized at $20^{\circ} \mathrm{C}$ by sitting drop vapour diffusion using $0.2 \mu \mathrm{L}$ protein solution $(30 \mathrm{mg} / \mathrm{mL}$ purified protein in Buffer $\mathrm{A})$ and $1 \mu \mathrm{L}$ of mother liquor $(1.44 \mathrm{M}$ lithium sulphate, $0.08 \mathrm{M}$ HEPES $\mathrm{pH} 7.5,0.02 \mathrm{M}$ sodium acetate $\mathrm{pH} 4.6,0.015 \mathrm{M}$ ammonium sulfate, $4 \%(\mathrm{w} / \mathrm{v})$ polyethylene glycol (PEG) 2000 monomethyl ether (MME)) with the addition of $1 \mathrm{mM}$ meropenem. The best diffracting YcbB crystal had the addition of a tetrapeptide (L-Ala-D-Glu- $m$ DAP-D-Ala) at $1 \mathrm{mM}$, though no density could be found for this ligand. E. coli PBP5 protein was crystallized at $20^{\circ} \mathrm{C}$ by sitting drop vapour diffusion using $0.2 \mu \mathrm{L}$ protein solution $(5 \mathrm{mg} / \mathrm{mL}$ purified protein in Buffer $\mathrm{A}$ ) and $1 \mu \mathrm{L}$ of mother liquor (0.1 M tris $\mathrm{pH} 7,7 \%$ PEG 400) with the addition of $1 \mathrm{mM}$ meropenem. X-ray diffraction data of YcbB-meropenemethylmercury and PBP5 was collected on Canadian Light Source beamline 08B1-1 using crystals flash-frozen in liquid nitrogen with the addition of $20 \%$ glycerol to the mother liquor. Data for native YcbB-meropenem was collected on Advanced Light Source beamline 501. For phasing of YcbB, a YcbB-meropenem crystal was soaked in mother liquor supplemented with $1 \mathrm{mM}$ ethylmercury phosphate for $30 \mathrm{~s}$. A single isomorphous replacement (SIR) experiment was carried out using the ethylmercury phosphate soaked crystal. All datasets were processed with $\mathrm{XDS}^{28}$, run manually or through Autoprocess ${ }^{29}$. For the SIR dataset, SHARP $^{30}$ was used for phasing, model building was performed by AutoBuild ${ }^{31}$ and refined using Phenix $^{32}$ and $\operatorname{Coot}^{33}$. For the native YcbB dataset and the PBP5 dataset, the structures were solved by molecular replacement using Phaser ${ }^{34}$. The SIR YcbB preliminary structure was used as the template for the YcbB structure and PDBID $3 \mathrm{MZF}$ was used to phase the PBP5 crystal. These structures were then refined using Phenix ${ }^{32}$ and $\operatorname{Coot}^{33}$. The capping loop region of $\mathrm{YcbB}$ was refined using densityguided iterative local refinement as implemented in Rosetta ${ }^{35}$. See Supplementary Table 1 for data collection and refinement statistics. See Supplementary Figs. 1 and 9 for example electron density.

Molecular dynamics simulations. A complete model for the meropenem acyl and apoenzyme forms of YcbB for molecular dynamics simulations was built from our structure of YcbB-meropenem acyl-enzyme complex with missing disordered loop regions built in using ModLoop ${ }^{36}$. This was then solvated and simulation input files were generated using Quick MD Simulator of the online CHARMM-GUI web service $^{37,38}$. Equilibration and production simulations were performed with GROMACS $5.1^{39}$, using the CHARMM36 all-atom force field ${ }^{40}$ to represent all protein, solvent and ions. Production simulations were run for $10 \mathrm{~ns}$. RMSD calculations were performed using the RMSD Visualizer Tool and HeatMapper of $\mathrm{VMD}^{41}$.

Impact of substitutions in YcbB on beta-lactam resistance. Beta-lactam resistance mediated by bypass the PBPs requires high-level production of the alarmone (p)ppGpp and of $\mathrm{YcbB}^{5}$. In this study, we used strain BW25113 $\Delta$ relA::Km ${ }^{\mathrm{R}}$ pKT8 $($ relA'), which allows the arabinose-inducible production of (p)ppGpp. The strain was transformed with the derivatives of pKT2 $(y c b B)$ for IPTG-inducible expression of $\mathrm{YcbB}$ and pf derivatives with amino acid substitutions. Primers used to clone YcbB with amino acid substitutions are listed in Supplementary Table 2. The phenotype was analyzed using the disk diffusion assay in BHI agar supplemented with $50 \mu \mathrm{M}$ IPTG, $1 \%$ arabinose, or both inducers, as previously described ${ }^{5}$. Disks were loaded with $10 \mu \mathrm{g}$ of mecillinam, $10 \mu \mathrm{g}$ of ampicillin, $30 \mu \mathrm{g}$ of ceftriaxone, 30 $\mu \mathrm{g}$ of tetracycline, $30 \mu \mathrm{g}$ of chloramphenicol, or $30 \mu \mathrm{g}$ of kanamycin. Incubation was performed at $37^{\circ} \mathrm{C}$ overnight. Resistance to beta-lactams $\left[\beta-l a c^{\mathrm{R}}\right]$ was defined as growth at the contact of disk containing ampicillin and a diameter $<17 \mathrm{~mm}$ for the inhibition zone around the disk containing ceftriaxone, as previously described $^{5}$. Susceptibility $\left[\beta-\mathrm{lac}^{\mathrm{S}}\right]$ was defined as diameters $>20 \mathrm{~mm}$ and $>35 \mathrm{~mm}$ for the inhibition zones around the disks containing ampicillin and ceftriaxone, respectively. We checked that resistance to ampicillin and ceftriaxone was dependent upon the presence of both inducers.

Reporting Summary. Further information on experimental design is available in the Nature Research Reporting Summary linked to this article.

\section{Data availability}

Atomic coordinates for the YcbB-meropenem and PBP5-meropenem models have been deposited in the protein data bank with accession codes 6 NTW and 6 NTZ respectively.

Received: 24 October 2018 Accepted: 13 March 2019

Published online: 23 April 2019

\section{References}

1. Sobhanifar, S., King, D. T. \& Strynadka, N. C. J. Fortifying the wall: synthesis, regulation and degradation of bacterial peptidoglycan. Curr. Opin. Struct. Biol. 23, 695-703 (2013).

2. Lovering, A. L., Safadi, S. S. \& Strynadka, N. C. J. Structural Perspective of Peptidoglycan Biosynthesis and Assembly. Annu. Rev. Biochem. 81, 451-478 (2012).

3. Caveney, N. A., Li, F. K. \& Strynadka, N. C. Enzyme structures of the bacterial peptidoglycan and wall teichoic acid biogenesis pathways. Curr. Opin. Struct. Biol. 53, 45-58 (2018)

4. King, D. T., Sobhanifar, S. \& Strynadka, N. C. J. One ring to rule them all: current trends in combating bacterial resistance to the $\beta$-lactams. Protein Sci. 25, 787-803 (2016).

5. Hugonnet, J.-E. et al. Factors essential for L,D-transpeptidase-mediated peptidoglycan cross-linking and $\beta$-lactam resistance inEscherichia coli. ELife 5, 1-22 (2016).

6. Triboulet, S. et al. Kinetic features of L,D-transpeptidase inactivation critical for $\beta$-lactam antibacterial activity. PLoS ONE 8, e67831 (2013).

7. Curtis, N. A., Orr, D., Ross, G. W. \& Boulton, M. G. Affinities of penicillins and cephalosporins for the penicillin-binding proteins of Escherichia coli K-12 and their antibacterial activity. Antimicrob. Agents Chemother. 16, 533-539 (1979).

8. Kumar, P. et al. Non-classical transpeptidases yield insight into new antibacterials. Nat. Chem. Biol. 13, 54-61 (2017).

9. Peters, K. et al. Copper inhibits peptidoglycan LD-transpeptidases suppressing $\beta$-lactam resistance due to bypass of penicillin-binding proteins. Proc. Natl Acad. Sci. USA 115, 10786-10791 (2018). 
10. Bianchet, M. A. et al. Structural insight into the inactivation of Mycobacterium tuberculosis non-classical transpeptidase LdtMt2by biapenem and tebipenem. BMC Biochem. 18, 1-14 (2017).

11. Maciejewska, B. et al. Modular endolysin of Burkholderia AP3 phage has the largest lysozyme-like catalytic subunit discovered to date and no catalytic aspartate residue. Sci. Rep. 7, 1-12 (2017).

12. Petersen, T. N., Brunak, S., von Heijne, G. \& Nielsen, H. SignalP 4.0: discriminating signal peptides from transmembrane regions. Nat. Methods 8, 785-786 (2011).

13. Bielnicki, J. et al. B. subtilis ykuD protein at $2.0 \AA$ resolution: Insights into the structure and function of a novel, ubiquitous family of bacterial enzymes. Proteins Struct. Funct. Genet. 62, 144-151 (2006).

14. Holm, L. \& Rosenström, P. Dali server: Conservation mapping in 3D. Nucleic Acids Res. 38, 545-549 (2010).

15. Fokine, A., Miroshnikov, K. A., Shneider, M. M., Mesyanzhinov, V. V. \& Rossmann, M. G. Structure of the bacteriophage $\varphi \mathrm{KZ}$ lytic transglycosylase gp144. J. Biol. Chem. 283, 7242-7250 (2008).

16. Biarrotte-Sorin, S. et al. Crystal Structure of a Novel $\beta$-Lactam-insensitive Peptidoglycan Transpeptidase. J. Mol. Biol. 359, 533-538 (2006).

17. Lecoq, L. et al. Structure of Enterococcus faecium 1, d -Transpeptidase Acylated by Ertapenem Provides Insight into the Inactivation Mechanism. Acs. Chem. Biol. 8, 1140-1146 (2013).

18. Gokulan, K., Khare, S., Cerniglia, C. E., Foley, S. L. \& Varughese, K. I. Structure and inhibitor specificity of L,D-Transpeptidase (LdtMt2) from mycobacterium tuberculosis and antibiotic resistance: calcium binding promotes dimer formation. Aaps. J. 20, 44 (2018).

19. Fakhar, Z. et al. Differential flap dynamics in l,d-transpeptidase 2 from Mycobacterium tuberculosis revealed by molecular dynamics. Mol. Biosyst. 13, 1223-1234 (2017).

20. Voss, N. R. \& Gerstein, M. 3V: Cavity, channel and cleft volume calculator and extractor. Nucleic Acids Res. 38, 555-562 (2010).

21. Erdemli, S. B. et al. Targeting the cell wall of Mycobacterium tuberculosis: structure and mechanism of L,D-transpeptidase 2. Structure 20, 2103-2115 (2012).

22. King, D. T., Wasney, G. A., Nosella, M., Fong, A. \& Strynadka, N. C. J. Structural insights into inhibition of Escherichia coli penicillin-binding protein 1B. J. Biol. Chem. 292, 979-993 (2017).

23. Alexander, J. A. N. et al. Structural and kinetic analysis of penicillin-binding protein 4 (PBP4)-mediated antibiotic resistance in Staphylococcus aureus. J. Biol. Chem. 4, jbc.RA118.004952 (2018).

24. Nicola, G., Tomberg, J., Pratt, R. F., Nicholas, R. A. \& Davies, C. Crystal structures of covalent complexes of $\beta$-Lactam antibiotics with escherichia coli penicillin-binding protein 5: toward an understanding of antibiotic specificity. Biochemistry 49, 8094-8104 (2010).

25. Birnbaum, J., Kahan, F. M., Kropp, H. \& MacDonald, J. S. Carbapenems, a new class of beta-lactam antibiotics. Discovery and development of imipenem/ cilastatin. Am. J. Med. 78, 3-21 (1985).

26. Pazos, M., Peters, K. \& Vollmer, W. Robust peptidoglycan growth by dynamic and variable multi-protein complexes. Curr. Opin. Microbiol. 36, 55-61 (2017).

27. Schanda, P. et al. Atomic model of a cell-wall cross-linking enzyme in complex with an intact bacterial peptidoglycan. J. Am. Chem. Soc. 136, 17852-17860 (2014).

28. Kabsch, W. XDS. Acta Crystallogr. D. Biol. Crystallogr. 66, 125-132 (2010).

29. Fodje, M. et al. 08B1-1: An automated beamline for macromolecular crystallography experiments at the Canadian Light Source. J. Synchrotron Radiat. 21, 633-637 (2014)

30. Bricogne, G., Vonrhein, C., Flensburg, C., Schiltz, M. \& Paciorek, W. Generation, representation and flow of phase information in structure determination: recent developments in and around SHARP 2.0. Acta Crystallogr. D. Biol. Crystallogr. 59, 2023-2030 (2003).

31. Terwilliger, T. C. et al. Iterative model building, structure refinement and density modification with the PHENIX AutoBuild wizard. Acta Crystallogr. D. Biol. Crystallogr. 64, 61-69 (2007).

32. Adams, P. D. et al. PHENIX: A comprehensive Python-based system for macromolecular structure solution. Acta Crystallogr. D. Biol. Crystallogr. 66, 213-221 (2010).

33. Emsley, P. \& Cowtan, K. Coot: Model-building tools for molecular graphics. Acta Crystallogr. Sect. D. Biol. Crystallogr. 60, 2126-2132 (2004).

34. McCoy, A. J. et al. Phaser crystallographic software. J. Appl. Crystallogr. 40, 658-674 (2007).

35. DiMaio, F. et al. Atomic-accuracy models from 4.5 - $\AA$ cryo-electron microscopy data with density-guided iterative local refinement. Nat. Methods 12, 361-365 (2015)

36. Fiser, A. \& Sali, A. ModLoop: automated modeling of loops in protein structures. Bioinformatics 19, 2500-2501 (2003).
37. Jo, S., Kim, T., Iyer, V. G. \& Im, W. CHARMM-GUI: a web-based graphical user interface for CHARMM. J. Comput. Chem. 29, 1859-1865 (2008)

38. Lee, J. et al. CHARMM-GUI input generator for NAMD, GROMACS, AMBER, OpenMM, and CHARMM/OpenMM simulations using the CHARMM36 additive force field. J. Chem. Theory Comput. 12, 405-413 (2016).

39. Abraham, M. J. et al. GROMACS: high performance molecular simulations through multi-level parallelism from laptops to supercomputers. SoftwareX 1-2, 19-25 (2015)

40. Best, R. B. et al. Optimization of the additive CHARMM all-atom protein force field targeting improved sampling of the backbone $\varphi, \psi$ and sidechain $\chi(1)$ and $\chi(2)$ dihedral angles. J. Chem. Theory Comput. 8, 3257-3273 (2012).

41. Humphrey, W., Dalke, A. \& Schulten, K. VMD: visual molecular dynamics. Mol. Graph. 14, 27-28 (1996).

\section{Acknowledgements}

We thank Michael Carlson and Franck Duong for the generous sample of activated SMA used for PBP1b solubilisation. We thank Jean-Pierre Simorre and Fred Rossell for advice on L,D-transpeptidases and protein-protein interactions, respectively. Research described in this paper was performed using beamline 08ID-1 at the Canadian Light Source and beamline 501 at the Advanced Light Source. The Canadian Light Source is supported by the Canada Foundation for Innovation, Natural Sciences and Engineering Research Council of Canada, the University of Saskatchewan, the Government of Saskatchewan, Western Economic Diversification Canada, the National Research Council Canada, and the Canadian Institutes of Health Research. This research also used resources of the Advanced Light Source, which is a DOE Office of Science User Facility under contract no. DE-AC02-05CH11231. This work was funded by operating grants to NCJS from JPIAMR-CIHR and the Howard Hughes International Senior Scholar program. This work was additionally supported by the project NAPCLI from the JPI AMR program to MA ( $N^{\circ}$ ANR-14-JAMR-0003-01). We also acknowledge infrastructure funding from the Canadian Foundation of Innovation and British Columbia Knowledge Development Fund. N.A.C. holds a Centre for Blood Research Graduate Award and NCJS is a Tier Canada Research Chair in Antibiotic Discovery.

\section{Author contributions}

N.A.C. contributed to conceptualization, methodology, investigation, analysis, writingoriginal draft, review, and editing. G.C. contributed to methodology and investigation. H. V. contributed to investigation and analysis. A.N. contributed to investigation. L.J.W. contributed to investigation and analysis. M.V. contributed to investigation. M.F. contributed to investigation and analysis. J.E.H. contributed to analysis and supervision. M. A. contributed to analysis and supervision. N.C.J.S. contributed to conceptualization, supervision, writing - review and editing, and funding acquisition.

\section{Additional information}

Supplementary Information accompanies this paper at https://doi.org/10.1038/s41467 019-09507-0.

Competing interests: The authors declare no competing interests.

Reprints and permission information is available online at http://npg.nature.com/ reprintsandpermissions/

Journal peer review information: Nature Communications thanks Anthony Clarke and Seok-Yong Lee for their contribution to the peer review of this work.

Publisher's note: Springer Nature remains neutral with regard to jurisdictional claims in published maps and institutional affiliations.

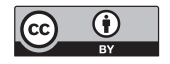

Open Access This article is licensed under a Creative Commons Attribution 4.0 International License, which permits use, sharing, adaptation, distribution and reproduction in any medium or format, as long as you give appropriate credit to the original author(s) and the source, provide a link to the Creative Commons license, and indicate if changes were made. The images or other third party material in this article are included in the article's Creative Commons license, unless indicated otherwise in a credit line to the material. If material is not included in the article's Creative Commons license and your intended use is not permitted by statutory regulation or exceeds the permitted use, you will need to obtain permission directly from the copyright holder. To view a copy of this license, visit http://creativecommons.org/ licenses/by/4.0/

(C) Crown 2019 